\title{
Features of Collagen Matrix Reconstructed with Proteodermatan sulfate from Pigskin Insoluble Collagen
}

\author{
Yoshihiro Nomura, Koji TaKahashi, Kunio ShIRAI \\ and Keizo WADA \\ Faculty of Agriculture, Tokyo Noko University, \\ Fuchu-shi, Tokyo 183, Japan
}

Received September 14, 1988

\begin{abstract}
Pigskin insoluble collagen was step-wise extracted with $0.5 \mathrm{M}$ and $4 \mathrm{M}$ guanidine hydrochloride (GuHCl) solution. Two proteoglycan preparations were obtained by DEAE-Toyopearl chromatography of the extracts, PG-I from $0.5 \mathrm{M}$ GuHCl extract and PG-II from $4 \mathrm{M}$ GuHCl extract. The proteoglycan preparations both contained at least two components having different mobilities on SDSPAGE $\left(M_{r}\right.$, about $60 \times 10^{3}$ and $\left.90 \times 10^{3}\right)$, and were identified as proteodermatan sulfate (PDS) by twodimensional electrophoresis on a cellulose acetate membrane. The two proteodermatan sulfates (PG-I and PG-II) accelerated the rate of the reconstruction of collagen matrix from neutral collagen solution, while the accelerating effect of PG-I was greater than that of PG-II. Electron microscopic observation showed that collagen matrices reconstructed with the PDSs consisted of thick fibrils with the banding pattern of native type and thin fibrils with an indistinct banding pattern. Collagen matrix reconstructed with addition of PDSs showed lower denaturation temperature than the control, as assessed by differential scanning calorimetry. These results suggest that collagen tissue-bound PDSs have a regulative function in the reconstruction of collagen matrix and its thermal stability.
\end{abstract}

We have been investigating the structural features of pigskin insoluble collagen especially with an aim for using ${ }^{1 \sim 6)}$ it as a biomaterial. In a series of these studies, it was found that components containing hexosamine, hexuronate, and non-collagenous proteins had remained in pigskin insoluble collagen which had been prepared with the most careful procedure. The components could be solubilized with calcium hydroxide ${ }^{7)}$ or magnesium chloride solution. ${ }^{8}$ These results suggest that the components, probably proteoglycans bound tightly to collagen tissue, take part in the morphology of collagen fibrils and stability of collagen matrix.

Proteodermatan sulfate (PDS) ${ }^{9)}$ and proteochondoroitin sulfate ${ }^{10)}$ are present in fresh pigskin, and the main component is PDS. ${ }^{11}$ Fujii et al., ${ }^{12)}$ Nakamura et al. ${ }^{13)}$ Damle et al., ${ }^{9)}$ and Miyamoto et al. ${ }^{14)}$ isolated PDSs from cattle hide, newborn calf skin, pigskin, and rat skin respectively. All these PDSs are much smaller $\left(M_{r}\right.$, about $\left.36 \sim 115 \times 10^{3}\right)$ than the well known chondroitin sulfate proteoglycans $\left(M_{r}>10^{6}\right)$ from cartilage and other connective tissues. Therefore, they can be classified as the small proteoglycan group. Since the proteoglycans described above have been extracted from skin containing soluble collagens, it is considered that the PDS preparations are mainly composed of PDS from soluble collagens, not IC.

It has been reported that PDS takes part in the reconstruction of the collagen matrix. Snowden et al. ${ }^{15)}$ showed that proteoglycan (probably PDS) from cartilage can accelerate the fibril formation of collagen and reduce the thermal stability of the reconstructed fibrils. However, Vogel et al. ${ }^{16)}$ showed that the PDS from bovine tendon reduced the rate of collagen matrix reconstruction. Scott et al. ${ }^{17,18)}$ concluded from electron microscopic observation using capromeronic blue stain that the PDS associated with collagen fibrils in skin at the location of the D periodical gap zones. However, little information is available on 
PDS bound tightly to collagenous tissue of pigskin in respect to its biochemical properties, especially the regulative function of collagen matrix reconstruction.

In this study, we isolated proteodermatan sulfate from purified pigskin insoluble collagen and investigated its effect on the rate of matrix reconstruction from collagen solution, the morphological features of the fibrils, and the thermal stability of the reconstructed collagen matrix.

\section{Materials and Methods}

1. Collagen preparations. Purified collagen preparations were prepared as in our previous paper $^{7)}$ as follows: insoluble collagen (IC) was prepared from fresh pigskin corium by extracting with $10 \% \mathrm{NaCl}$ solution, acetoneether, $1 / 15 \mathrm{M}$ phosphate buffer at $\mathrm{pH} 8.0$ and $0.5 \mathrm{M}$ acetic acid solution containing $0.5 \mathrm{M} \mathrm{NaCl}$. Neutral salt soluble collagen (NSC) was obtained from $1 / 15 \mathrm{M}$ phosphate extracts by salt prcipitation. After purification, NSC was used for the experiment of collagen matrix reconstruction.

2. Extraction of uronate-containing components. To choose the extracting solvent, uronate-containing components bound to IC were step-wise extracted with magnesium chloride $(0.1 \mathrm{M}, 1.0 \mathrm{M}$ and $3.0 \mathrm{M})$ or guanidine hydrochloride $(0.5 \mathrm{M}$ and $4.0 \mathrm{M})$ solutions in $0.02 \mathrm{M}$ Trishydrochloride buffer at $\mathrm{pH} 7.0$ for $48 \mathrm{hr}$ at $4^{\circ} \mathrm{C}$, using ten times the solvent volume based on IC weight. These extracts were centrifuged at $8000 \mathrm{rpm}$. The supernatants were dialyzed against distilled water. Precipitates during dialysis were separated by centrifugation and lyophilized. The extractability of uronate-containing components with guanidine hydrochloride solution, was better than that with magnesium chloride solution. Then $0.5 \mathrm{~m}$ guanidine extracts (Ext-I) and $4.0 \mathrm{M}$ guanidine extracts (Ext-II) were used for separating proteoglycan.

3. Isolation of proteoglycan by ion-exchange chromatography. Proteoglycans were isolated from Ext-I and Ext-II on a DEAE-Toyopearl $650 \mathrm{~S}$ column under the following conditions by the method of Antonopoulos et al. ${ }^{19)}$; column size, $2.6 \times 30 \mathrm{~cm}$; elution, linear gradient of sodium chloride concentration from 0 to $1.0 \mathrm{M}$ in $0.05 \mathrm{M}$ Trishydrochloride buffer at $\mathrm{pH} 7.0$ containing $7 \mathrm{M}$ urea deionized by ion-exchange resins; flow rate, $120 \mathrm{mi} / \mathrm{hr}$; temperature, $25^{\circ} \mathrm{C}$; detection, for protein, absorbance at $230 \mathrm{~nm}$ and for uronate, the sulfuric acid-carbazole method, ${ }^{20)}$ fractions, $10 \mathrm{ml} /$ tube. The chromatographic fractions containing both noncollagenous proteins and hexuronate were collected, dialyzed against distilled water, and lyophilized.
4. Two-dimensional electrophoresis on a cellulose acetate membrane. Glycosaminoglycan chains were separated by digesting proteoglycan with Pronase after alkaline cleavage by the method of Ninomiya et $a .^{21)}$ Glycosaminoglycan was identified by two-dimensional electrophoresis on cellulose acetate membrane by the method of Hata et al. $^{22)}$

5. Preparation of core protein. By the method of Oike $e t$ $a l .{ }^{23)}$ the core protein was separated by digesting PDS with chondroitinase $\mathrm{ABC}$ in the presence of protease inhibitors at $37^{\circ} \mathrm{C}$ for $40 \mathrm{~min}$, then precipitated with ethanol.

6. SDS-polyacrylamide gel electrophoresis (SDS$P A G E$ ). The chromatographic fractions, PDS and its components, were heated in electrophoretic buffer containing $1 \% \mathrm{SDS}$ at $100^{\circ} \mathrm{C}$ for $5 \mathrm{~min}$ and electrophoresed by SDS-PAGE by the method of Weber and Osborn, ${ }^{24)}$ using $5 \%$ polyacrylamide gel. Protein bands and glycosaminoglycan bands were stained with $0.12 \%$ Coomassie Brilliant Blue and $0.5 \%$ Alcian Blue, respectively. Coincidental stains of Coomassie Brilliant Blue and Alcian Blue at the same migration point on SDS-PAGE was taken as evidence of proteoglycan.

7. Reconstruction of collagen matrix. Neutral salt soluble collagen was dissolved in $0.5 \mathrm{M}$ acetic acid at $4^{\circ} \mathrm{C}$ to give a concentration of about $1 \mathrm{mg} / \mathrm{ml}$ and dialyzed against $1 / 15 \mathrm{M}$ phosphate buffer at $\mathrm{pH} 7.0$ before centrifugation at $18000 \mathrm{rpm}$ for $30 \mathrm{~min}$. After adjusting the concentration to $0.6 \mathrm{mg} / \mathrm{ml}$, PDS dissolved in the same phosphate buffer was added to give the following final conditions: collagen conc., $0.4 \mathrm{mg} / \mathrm{ml}$; PDS/collagen ratio, $1: 50$ or $1: 500$ (calculated as the weight ratio of glucuronic acid lactone to collagen). The solution was warmed to $30^{\circ} \mathrm{C}$ to initiate matrix reconstruction, and monitored by the absorbance at $310 \mathrm{~nm}$. After $24 \mathrm{hr}$, the amount of reconstructed collagen matrix was measured from the concentration of collagen in the supernatant centrifuged at $18000 \mathrm{rpm}$ for $30 \mathrm{~min}$.

8. Electron microscopic observation. The reconstructed collagen matrix was observed with a scanning electron microscope (SEM; JOEL JSM-U3) or transmission electron microscope (TEM; JOEL JEM-100C). Collagen matrix was collected by centrifugation at $3000 \mathrm{rpm}$, washed thrice with $1 / 15 \mathrm{~m}$ phosphate buffer at $\mathrm{pH} 7.0$ and fixed with $2 \%$ glutaraldehyde. Specimens for SEM observation were dehydrated with alcohol, dried by critical point drying with carbon dioxide, and coated with gold by ionsputter. Specimens for TEM observation were positively stained with $1 \%$ uranyl acetate and $1 \%$ phosphotungstic acid. Electron accelerators for SEM and TEM were operated at $15 \mathrm{kV}$ and $80 \mathrm{kV}$, respectively.

9. Differential scanning calorimetry (DSC). DSC of 
reconstructed collagen matrix was done using a DSC apparatus (Seiko SSC-560U) coupled with thermal analysis data system 560 DSIII as described previously. ${ }^{25)}$ The sample quantity in the cell was measured by the microbiuret method ${ }^{26)}$ dissolving it after DSC. From DSC measurements, the denaturation temperatures [onset temp. $\left(T_{\mathrm{o}}\right)$, peak temp. $\left(T_{\mathrm{p}}\right)$, and recovery temp. $\left.\left(T_{\mathrm{r}}\right)\right]$ and the denaturation enthalpy were recorded as characteristics of the heat-denaturation process.

10. Analytical methods. Uronic acid and proteins were measured by the sulfuric acid-carbazole method ${ }^{20)}$ and microbiuret method, ${ }^{26)}$ respectively.

\section{Results and Discussion}

1. Extraction of hexuronate-containing components from pigskin insoluble collagen

Proteoglycans from dermis are extracted by urea, ${ }^{27)}$ sodium chloride, ${ }^{13)}$ guanidine hydrochloride, ${ }^{9,14)}$ and magnesium chloride solutions. ${ }^{12,28)}$ In this experiment, we compared the effectiveness in step-wise extractions of proteoglycan with magnesium chloride and guanidine hydrochloride. The yield and the chemical composition of those extracts are shown in Table I. In the case of extraction with magnesium chloride solution, the yield at $1.0 \mathrm{M}$ was maximum, and the ratio of hexuronate to protein of the extract was maximum, 1/71. On the other hand, the hexuronate yield of extracts and the percent of hexuronate to protein in the guanidine hydrochloride solution were higher than that of magnesium chloride. The yield of $4 \mathrm{M}$ guanidine hydrochloride extracts was about $3 \%$. The hexuronate content of pigskin insoluble collagen was $0.5 \%(\mathrm{w} / \mathrm{w})$. The total hexuronates recovered in $0.5 \mathrm{M}$ and $4.0 \mathrm{M} \mathrm{GuHCl}$ extracts were $6.8 \%$ and $6.2 \%$, respectively, of that of IC. Electrophoretic migration patterns of $\mathrm{GuHCl}$ extracts are shown in Fig. 1. It was recognized in both extracts that there were two kinds of band stained with Coomassie Brilliant Blue, that is, bands coinciding with collagen chains and non-collagenous protein with faster migration than collagen chains. Furthermore, the positive staining with Alcian Blue of the non-

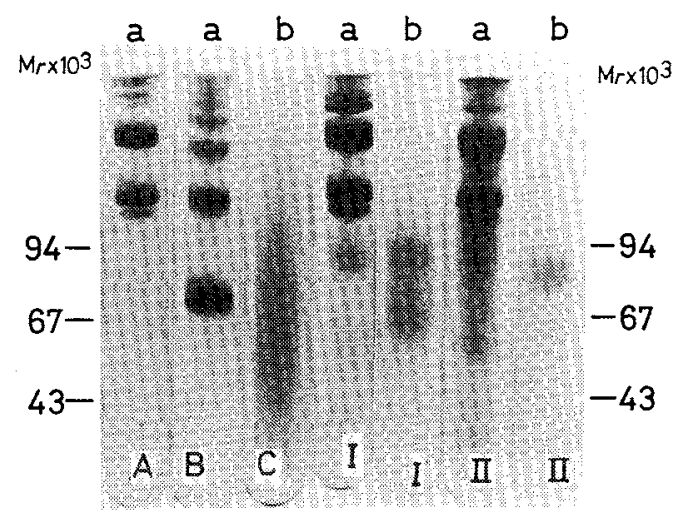

Fig. 1. SDS-Polyacrylamide Gel Electrophoresis of Guanidine Hydrochloride-extracts.

Samples extracted step-wise with $0.5 \mathrm{M}$ and $4.0 \mathrm{M}$ guanidine hydrochloride solutions were electrophoresed on $5 \%$ SDS-polyacrylamide gel under non-reducing conditions. a, stained with Coomassie Brilliant Blue; b, stained with Alcian Blue. A, acid soluble collagen; B, bovine serum albumin; $C$, chondroitin 6-sulfate; I, $0.5 \mathrm{M}$ guanidine hydrochloride extract; II, $4.0 \mathrm{M}$ guanidine hydrochloride extract.

Table I. Step-wise Extractions with Magnesium Chloride and Guanidine Hydrochloride SOlution and Chemical Composition of the Extracts

\begin{tabular}{|c|c|c|c|c|}
\hline \multirow{2}{*}{ Solvent } & \multirow{2}{*}{$\begin{array}{c}\text { Yield of } \\
\text { extraction from } \\
\text { insoluble collagen } \\
(\%)^{a}\end{array}$} & \multicolumn{3}{|c|}{ Chemical composition of extracts } \\
\hline & & $\begin{array}{c}\text { Protein } \\
(\%)^{b}\end{array}$ & $\begin{array}{c}\text { Uronic acid } \\
\qquad(\%)^{b}\end{array}$ & $\begin{array}{l}\text { Uronic acid/ } \\
\text { Protein ratio }\end{array}$ \\
\hline $0.1 \mathrm{M} \mathrm{MgCl}{ }_{2}$ & 1.3 & 70.6 & 0.14 & $1 / 504$ \\
\hline $1.0 \mathrm{M} \mathrm{MgCl}_{2}$ & 1.6 & 98.9 & 0.7 & $1 / 141$ \\
\hline $3.0 \mathrm{M} \mathrm{MgCl}_{2}$ & 0.5 & 100 & 0.3 & $1 / 333$ \\
\hline $0.5 \mathrm{M} \mathrm{GuHCl}$ & 1.3 & 67.9 & 1.3 & $1 / 52$ \\
\hline $4.0 \mathrm{M} \mathrm{GuHCl}$ & 2.6 & 82.8 & 0.6 & $1 / 138$ \\
\hline
\end{tabular}

a Extracts were expressed as percent $(\%)$ of insoluble collagen.

$b$ The protein and uronic acid of extracts were expressed as percent $(\%)$ of extract. 
collagenous protein band proved the existence of glycosaminoglycan. Electrophoretic migration patterns of magnesium chloride extracts were similar to that of $\mathrm{GuHCl}$ extracts except that the Alcian Blue stain of the former extracts was somewhat weaker than the latter. We investigated the proteoglycan from $\mathrm{GuHCl}$ extracts in detail.

\section{Isolation of proteoglycan}

To extract different proteoglycans from pu-

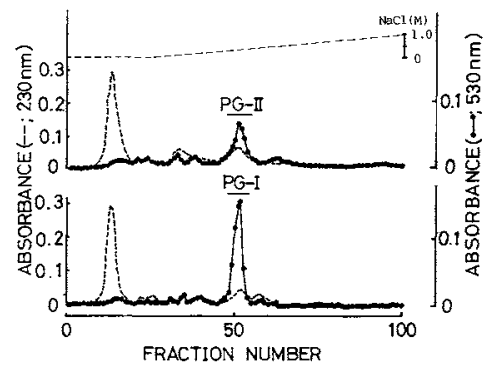

Fig. 2. Ion-exchange Chromatography of $0.5 \mathrm{M} \mathrm{GuHCl}$ (Ext-I) and $4.0 \mathrm{M} \mathrm{GuHCl}$ (Ext-II) Extracts from Pigskin Insoluble Collagen.

The extracts were dissolved in $7 \mathrm{M}$ urea $/ 0.05 \mathrm{~mm}$ Tris$\mathrm{HCl}$ buffer at $\mathrm{pH} 7.0$, and put on a column $(2.6 \times$ $30 \mathrm{~cm}$ ) of DEAE-Toyopearl $650 \mathrm{~S}$ after equilibrated with the same buffer. A linear gradient of sodium chloride concentration $(0 \sim 1 \mathrm{M})$ was started at tube No. 25. A flow rate of $120 \mathrm{ml} / \mathrm{hr}$ was maintained, and each fraction was analyzed for uronic acid by the carbazole reaction (solid line) and protein content (broken line). Top panel, Ext-II; Bottom panel, Ext-I. Fractions barred were collected as proteoglycan (PG-I or PG-II). rified pigskin insoluble collagen, the step-wise extraction was done with $0.5 \mathrm{M}$ and $4.0 \mathrm{M}$ guanidine hydrochloride in $0.02 \mathrm{M}$ Tris-hydrochloride buffer at $\mathrm{pH}$ 7.0. These extracts were named as Ext-I and Ext-II, respectively. Ion-exchange chromatography of Ext-I and Ext-II was carried out on a column of DEAEToyopearl 650S. The elution patterns are shown in Fig. 2. The elution pattern monitored at $230 \mathrm{~nm}$ indicated a strong peak at passing fractions and two small peaks at the fractions eluted at $0.3 \mathrm{M}$ and $0.5 \mathrm{M}$ sodium chloride concentrations. Passing fractions were con-

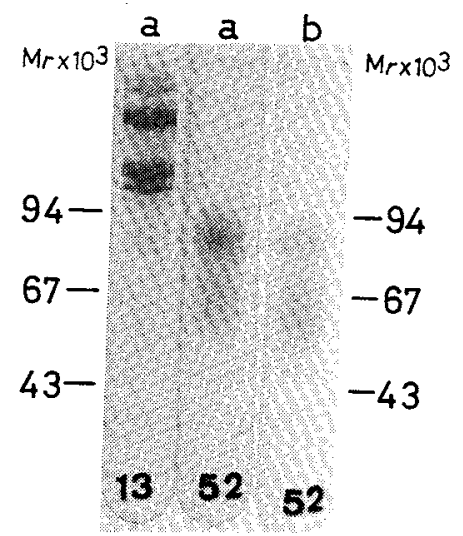

Fig. 3. SDS-Polyacrylamide Gel Electrophoresis of Ionexchange Chromatographic Fractions from Ext-I.

a, stained with Coomassie Brilliant Blue; $b$, satained with Alcian Blue; 13, passing fraction; 52, fraction eluted at $0.5 \mathrm{M}$ sodium chloride. For analytical conditions, see in Fig. 1.

Table II. Chemical Compositions of Proteoglycans, and Their Influences on Collagen Matrix Reconstruction

\begin{tabular}{|c|c|c|c|c|c|}
\hline \multirow{2}{*}{ Proteoglycan } & \multicolumn{2}{|c|}{ Chemical composition } & \multicolumn{3}{|c|}{$\begin{array}{l}\text { Time required for the maximum velocity of } \\
\text { matrix reconstruction }\left(T_{\max }, \min \right)\end{array}$} \\
\hline & $\begin{array}{c}\text { Protein } \\
\text { content }(\%)\end{array}$ & $\begin{array}{l}\text { Uronic acid } \\
\text { content }(\%)\end{array}$ & Control & $1 / 500$ & $1 / 50$ \\
\hline PG-I & 47.2 & 8.1 & $\begin{array}{c}46 \\
(75.5)\end{array}$ & $\begin{array}{c}14 \\
(81.4)\end{array}$ & $\overline{(86.5)}$ \\
\hline PG-II & 53.3 & 6.6 & $\begin{array}{c}46 \\
(75.5)\end{array}$ & $\begin{array}{c}3,54 \\
(87.4)\end{array}$ & $\frac{-}{(73.0)}$ \\
\hline
\end{tabular}

PG-I, PG-II: See Fig. 2.

For procedures of collagen matrix reconstruction, see Fig. 6 .

( ): Percent of reconstructed collagen matrix.

-: It is difficult to trace the absorbance change. 
firmed to be collagen (Fr. No. 13 in Fig. 3) by SDS-PAGE. The fractions eluted at $0.5 \mathrm{M}$ sodium chloride were collected, because they indicated relatively strong absorption based on uronate as well as non-collagenous protein, confirmed by SDS-PAGE (shown in Fig. 3). Almost the same SDS-PAGE patterns were obtained for Ext-II (data not shown). These fractions contained two proteinous components with different mobilities. Since both proteinous components indicated stains of glycosaminoglycan at the same migration position, it is considered that there are two kinds of proteoglycans in this fraction. The faster component in mobility had a somewhat stronger stain of glycosaminoglycan and weaker stain of protein than those of the slower one. These fractions eluted at $0.5 \mathrm{M}$ so-

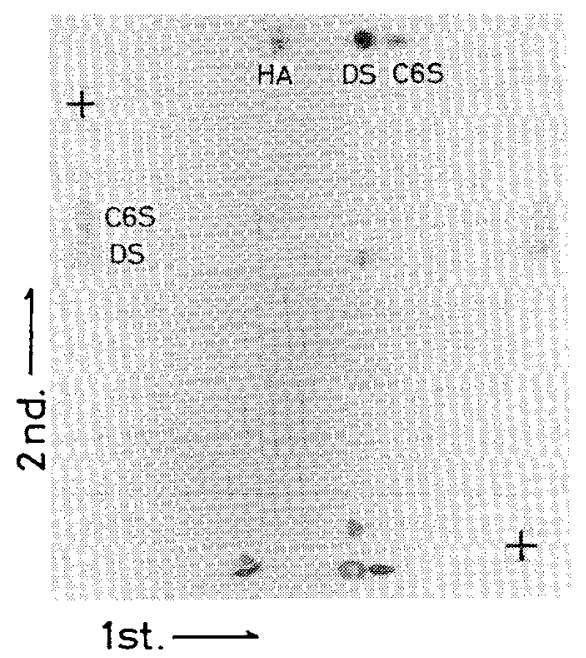

Fig. 4. Two-dimensional Electrophoresis of Glycosaminoglycan of Proteoglycan on a Cellulose Acetate Membrane.

The sample was prepared as described under Materials and Methods. Standard mixture is composed of hyaluronic acid (HA), chondoroitin-6 sulfate (Ch-6S), and dermatan sulfate (DS). Glycosaminoglycan standards for the run in the first dimension are Ch-6S, DS, and HA and in the second dimension are $\mathrm{Ch}-6 \mathrm{~S}$ and $\mathrm{DS}$, respectively. $\mathrm{Mi}$ grating directions are marked with arrows. Electrophoretic conditions; $0.1 \mathrm{M}$ pyridine $/ 0.47 \mathrm{M}$ formic acid, $\mathrm{pH} 3$, at $1 \mathrm{~mA} / \mathrm{cm}$ for $1.5 \mathrm{hr}$ in the first dimension and $0.1 \mathrm{M}$ barium acetate, $\mathrm{pH} 8$, at $1 \mathrm{~mA} / \mathrm{cm}$ for $4.5 \mathrm{hr}$ in the second. Glycosaminoglycan was stained with Alcian Blue. dium chloride in Ext-I and Ext-II were named as PG-I and PG-II, respectively, and used as proteoglycan preparations in the following experiments. The chemical compositions of PG-I and PG-II were shown in Table II. Since the uronate content of PG-II was about $30 \%$ lower than that of PG-I, it is considered that PG-I and PG-II are different.

The relative molecular weight $\left(M_{r}\right)$ of the two kinds of proteoglycans in PG-I preparations were estimated to be about $60 \times 10^{3}$ and $90 \times 10^{3}$, based on the mobility on SDS-PAGE (Fig. 3). The SDS-PAGE migration pattern of PG-II was the same as that of PG-I. The glycosaminoglycan chain of these proteoglycans were identified as dermatan sulfate by two-dimensional electrophoresis on a cellulose acetate membrane after Pronase digestion of the proteoglycan (Fig. 4). The mobility of the core protein-free glycosaminoglycan chains corresponds to $M_{r} 20 \sim 52 \times 10^{3}$ (as globular protein) on SDS-PAGE (Fig. 5.) Therefore, we concluded that both of the proteoglycans are proteodermatan sulfates. The SDS-PAGE pat-

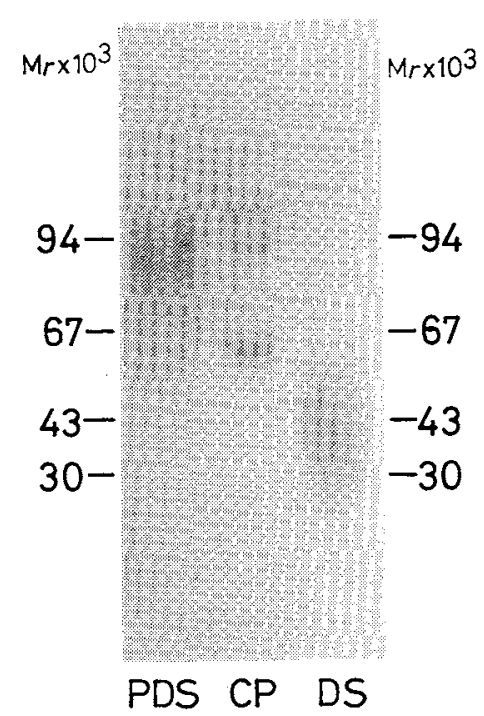

Fig. 5. SDS-Polyacrylamide Gel Electrophoresis of PGI, Glycosaminoglycan and Core Protein.

Samples were prepared as described under Materials and Methods. The gel was run under reducing conditions and was double stained with Coomassie Brilliant Blue and Alcian Blue. PDS, PG-I; CP, core protein; DS, dermatan sulfate side chain. Analytical conditions, see Fig. 1. 
tern of chondroitinase ABC-treated core molecule from PG-I is shown in Fig. 5. The core molecule gave one diffuse band with $M_{r}=$ $55 \times 10^{3}$ (two bands near $94 \times 10^{3}$ represent proteins in the chondroitinase $\mathrm{ABC}$ preparation). This means that core molecules from two kinds of PDSs of PG-I are the same molecular weight. The $M_{r}$ values somewhat larger than that of the corresponding core molecule $\left(M_{r}, 44 \times 10^{3}\right)$ from pigskin $\operatorname{PDS}\left(M_{r}\right.$, $70 \times 10^{3}$ ) reported by Damle et al. ${ }^{93}$ Further investigations are required to find whether these proteoglycans represent different molecular species.

\section{Reconstruction of collagen matrix with PDS}

Changes of the absorbance of collagen solution containing PDS at the ratio of 1:500 based on the uronic acid content to the amount of collagen (weight ratio of PDS/ collagen is about $1 / 100$ ) were measured to investigate the effect of PDS (PG-I and PGII) on collagen matrix reconstruction. $\mathrm{Ab}$ sorbance/incubation time curves, that is matrix reconstruction curves, are shown in Fig. 6 . The matrix reconstruction curves ordinarily have three or four steps. The first step is the lag phase without absorbance change, called the nucleation time. The second step is the growth phase with the initiation of increase in absorbance. The third step is the maturity phase with a rapid increase in absorbance accompanied by the formation of a threedimensional network of collagen matrix. The fourth step is the stationary phase. Derivatives of these curves give characteristics of absorbance curves at the point of the highest slope $\left(T_{\max }\right)$ corresponding to the parameter of the third step. Values of $T_{\max }$ were recorded in Table II. In case of no addition of PDS, the control $T_{\max }$ was $46 \mathrm{~min}$. With the addition of $1 / 500$ of PG-I, $T_{\max }$ decreased to $1 / 3$ of the control, indicating the acceleration of the rate of matrix reconstruction due to fast aggregation of collagen molecules. Upon the addition of 1/500 of PG-II, as shown in Fig. 6, an inflection point with low absorbance appeared at a very early incubation time, and the matrix

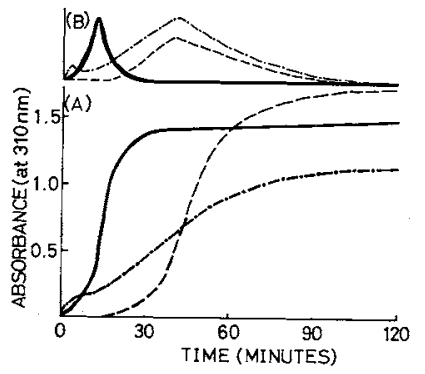

Fig. 6. Reconstruction of Collagen Matrix with Proteoglycan.

Proteoglycan $(0.8 \mu \mathrm{g} / \mathrm{ml})$ was incubated with collagen solution $(400 \mu \mathrm{g} / \mathrm{ml})$ at $30^{\circ} \mathrm{C}$. Matrix reconstruction was monitored by the absorbance at $310 \mathrm{~nm}$. - , PG-I; -.-, PG-II; ----, control. (A): Changes of absorbance during collagen matrix reconstruction. (B): Derivative curves of the curves in panel $A$.

reconstruction curve indicated two phases (minor phase and major phase), while $T_{\max }$ of the major phase was little different from the control. When the additional ratio of either PG-I or PG-II increased to $1 / 50$, it was difficult to trace the absorbance change due to too rapid aggregation of collagen molecules. This means that either PG-I or PG-II accelerated essentially collagen matrix reconstruction, while the effect of PG-I appeared at a much lower concentration then that of PG-II. We also noted that with the same weight bases, the accelerating effect of commercial dermatan sulfate on the rate of reconstruction of collagen matrix was greater than that of PG-I or PG-II (data not shown). From this finding, the higher content of glycosaminoglycan of PG-I than that of PG-II as seen in Table II, may be attributable to the difference in the acceleration effect of matrix reconstruction. When PDSs were added at the ratio of $1: 50$, the amount of reconstructed matrix was $80 \sim 90 \%$ as compared with $76 \%$ in the control. Uronate was not detected in the supernatant centrifuged after the reconstruction of the collagen matrix. This suggests that the PDSs added were incorporated into reconstructed collagen matrix. 
4. Electron microscopic observation of reconstructed collagen matrix

Electron micrographs of collagen matrix reconstructed are shown in Fig. 7. SEM of the control matrix demonstrated thick fibrils (mean diameter, about $170 \mathrm{~nm}$ ) forming a welldeveloped network structure. TEM of the control matrix demonstrated native type fibrils with cross-striated structure of a period of $64 \mathrm{~nm}$ and subbands of 10 lines in one period. Upon the addition of PG-I at a ratio of $1: 500$, predominant thick fibrils similar to the control and thin fibrils (mean diameter, about $50 \mathrm{~nm}$ ) as a minor filler among the predominant fibrils were observed with SEM. TEM demonstrated mainly native type band patterns on thick fibrils similar to control fibrils, indistinct band patterns on thin fibrils and FLS type band patterns on a very small amont of thin fibrils. It appeared that the indistinct thin fibrils corresponded to the thin filler fibrils observed on SEM. When the addition of PG-I was in-
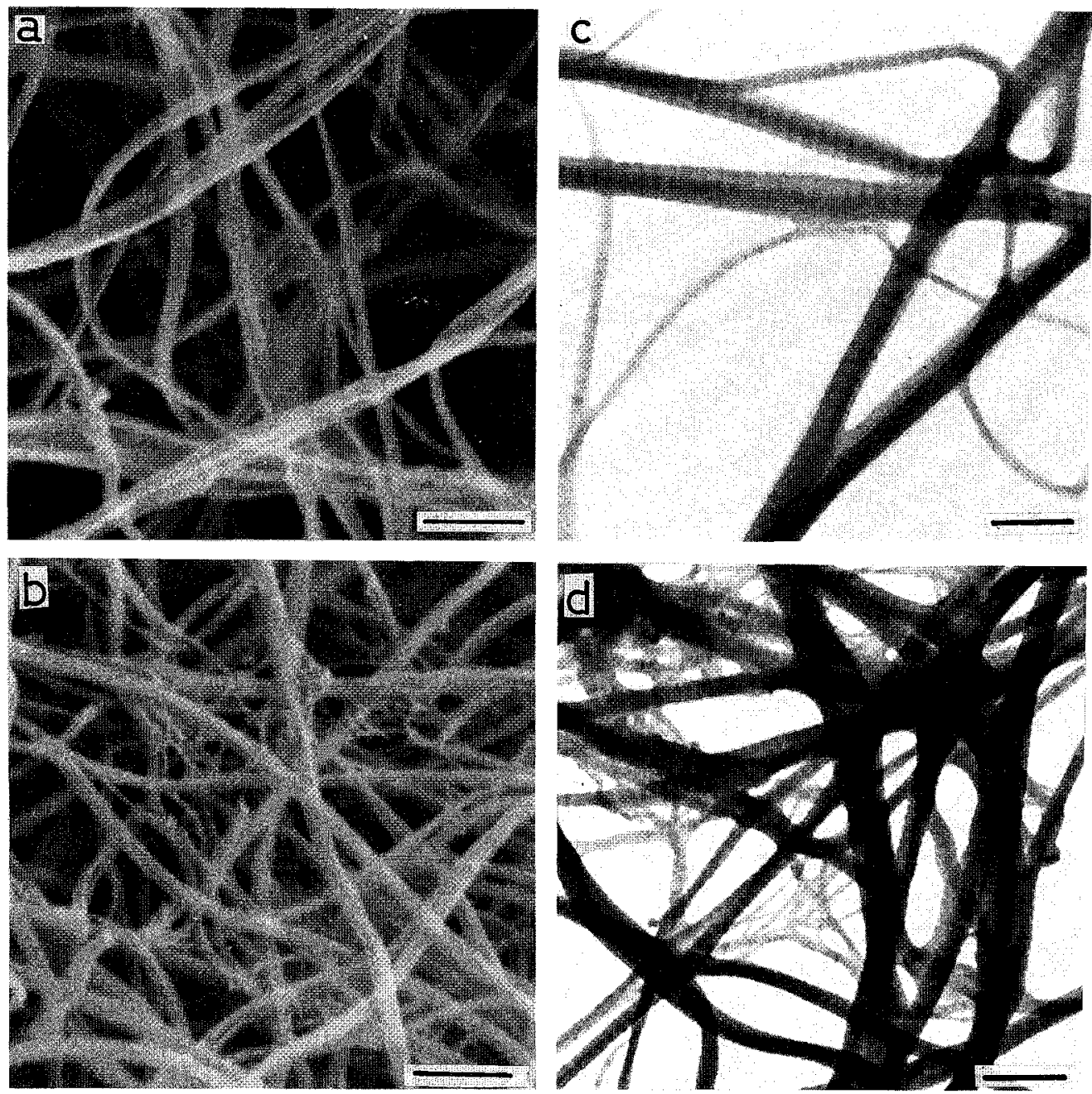

Fig. 7. Electron Micrographs of Collagen Matrix Reconstructed with PG-I.

Collagen matrix was reconstructed with or without the addition of PG-I at the ratio of $1: 500$, and processed for electron microscopic visualization as described under Materials and Methods. a and c, control matrix; $b$ and d, collagen matrix reconstructed with PG-I; $a$ and b, SEM observations (bar indicates $2000 \mathrm{~nm}$ ); $\mathrm{c}$ and d, TEM observation (bar indicates $400 \mathrm{~nm}$ ). 
creased to the ratio of $1: 50$, the proportion of thin fibrils increased. The addition of PG-II also produced thick fibrils of native type banding pattern, thin fibrils with indistinct banding pattern, and a small amount of FLS type fibrils. The effects of PG-I and PG-II on the reconstruction of collagen matrix were similar. The addition of commercial chondroitin 6sulfate $(\mathrm{Ch}-6 \mathrm{~S})$ instead of the PDSs produced a substantial amount of the FLS type fibrils. The tendency of FLS type fibril formation for PG-I or PG-II was not strong compared with Ch-6S.

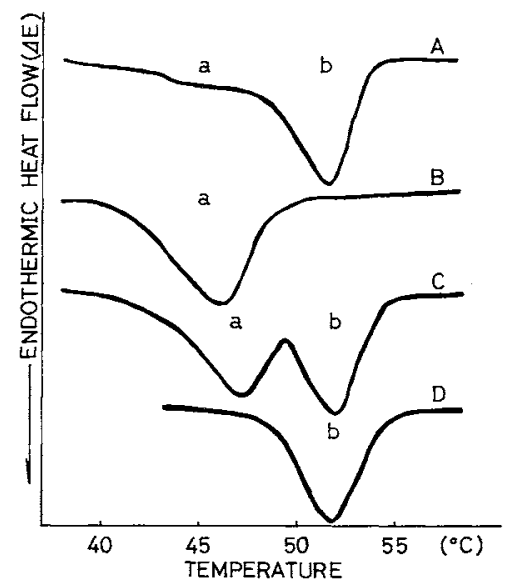

Fig. 8. Differential Scanning Calorimetry of Reconstructed Collagen Matrix with Proteoglycans.

A: The additional ratio of $\mathrm{PG}-\mathrm{II}, 1 / 500$. B: The additional ratio of $\mathrm{PG}-\mathrm{I}, 1 / 50$. C: The additional ratio of $\mathrm{PG}-\mathrm{I}, 1 / 500$. D: Control. Analytical conditions are described under Materials and Methods. Peak a, low temperature peak; peak $b$, high temperature peak.
Furthermore, the effects of PDS on induction of the indistinct band pattern were essentially different from those of Ch-6S.

Scott et al. ${ }^{17,18)}$ showed that PDS associated with collagen fibrils at the location of the D periodical gap zones. In this study, it is difficult to elucidate the binding site of proteoglycan to collagen. However, these observations, coupled with our finding that the PDSs isolated from collagen bundles were incorporated in to the collagen matrix, suggest that the PDSs are important in the formation of collagen fibrils; e.g. facilitation of the association (and/or nucleation) of collagen molecules and regulation of the aggregation of microfibrils.

From these results, it is considered that the effect of PDS bound to collagenous tissue is different from that of glycosaminoglycan on the reconstruction rate of collagen matrix and the rearrangement of collagen molecules.

\section{Thermal denaturation behavior of recon- structed collagen matrix}

DSC curves of collagen matrices reconstructed with PG-I or PG-II are shown in Fig. 8 , and thermal denaturation temperatures and denaturation enthalpies were summarized in Table III. Thermal denaturation of the control matrix occurred in a region from about $48^{\circ} \mathrm{C}$ to $56^{\circ} \mathrm{C}$, and indicated a sharp single peak, which was much higher than that of collagen molecules (from about $37^{\circ} \mathrm{C}$ to $46^{\circ} \mathrm{C}$ ) in the solution. ${ }^{29,30)}$ These DSC curves evidently in-

Table III. Denaturation Temperatures and Denaturation Enthalpies of Collagen Matrices Reconstructed with the Addition of Proteoglycans

\begin{tabular}{|c|c|c|c|c|c|c|c|}
\hline \multirow{3}{*}{ Collagen matrix } & \multicolumn{6}{|c|}{ Denaturation temperature $\left({ }^{\circ} \mathrm{C}\right)$} & \multirow{3}{*}{$\begin{array}{c}\text { Denaturation } \\
\text { enthalpy } \\
(\mathrm{ml} / \mathrm{mg})\end{array}$} \\
\hline & \multicolumn{3}{|c|}{ Peak a } & \multicolumn{3}{|c|}{ Peak b } & \\
\hline & $T_{\mathrm{o}}$ & $T_{\mathrm{p}}$ & $T_{\mathrm{x}}$ & $T_{\mathrm{o}}$ & $T_{\mathrm{p}}$ & $T_{\mathrm{r}}$ & \\
\hline Control & & & & 48.4 & 51.8 & 55.8 & 46.0 \\
\hline PG-I $(1 / 500)$ & 42.7 & 46.8 & & 49.2 & 51.9 & 55.3 & 46.3 \\
\hline PG-I $(1 / 50)$ & 41.7 & 46.0 & 51.5 & & & & 52.5 \\
\hline PG-II (1/500) & 41.1 & & & 48.6 & 51.5 & 55.4 & 50.2 \\
\hline
\end{tabular}

( ): Additional ratio of proteoglycan based on collagen.

For peak a and b, see Fig. 6: $T_{0}$, onset temperature of DSC curve; $T_{\mathrm{p}}$, peak temperature of DSC curve; $T_{\mathrm{r}}$, recovery temperature of DSC curve. 
dicate a thermal denaturation behavior of collagen forming a fibril network, not of dispersed molecules. The matrix with PG-I added at the ratio of $1: 500$ indicated two endothermic peaks (named peak a and peak $b$ around 47 and $52^{\circ} \mathrm{C}$ respectively) unlike the control. The peak $b$ of the high temperature region at about $52^{\circ} \mathrm{C}$ corresponds to the endothermic peak of the control. An increase in the addition amount of PG-I to 1/50 caused the disappearance of peak $b$ and the expansion of peak a at about $47^{\circ} \mathrm{C}$, meaning the formation of a poorly stabilized macrostructure of collagen. Since DSC can detect the difference of intermolecular cohesion in the macrostructure as the difference of thermal denaturation behavior, the data on the denaturation temperature suggest that PDS renders the collagen matrix rather weak in intermolecular cohesion. These results indicate that PG-I promotes the reconstruction of a less cohesive network of thin fibrils. Wallace et al. ${ }^{31)}$ suggested that the very thin fibrils from an acid soluble collagen solution denaturated below $50^{\circ} \mathrm{C}$, consistent with our finding. McPherson et al. ${ }^{32)}$ recognized that the addition of heparin to collagen fibrillar suspensions promotes the growth in fibril diameter and make fibrils grow to a higher stability on DSC. However, since this experiment investigated the influence of heparin on a collagen fibril suspension, not a collagen solution, their results may not directly related to fibrogenesis. The DSC curve of the collagen matrix reconstructed with $\mathrm{PG}-\mathrm{II}$ was similar to the case of PG-I, indicating a small shoulder linked to peak b. On the other hand, it seems that there is no great difference in the denaturation enthalpy among different matrices.

\section{Conclusion}

Two kinds of proteoglycans were extracted with $0.5 \mathrm{M}$ and $4.0 \mathrm{M}$ guanidine hydrochloride solution from purified pigskin insoluble collagen (PG-I from $0.5 \mathrm{M} \mathrm{GuHCl}$ extracts and PG-II from 4.0 M GuHCl extracts). Each proteoglycan preparation had two bands on SDS-
PAGE pattern $\left(M_{r}=60 \times 10^{3}, 90 \times 10^{3}\right)$ and was identified as dermatan sulfate proteoglycan containing a core molecule of $M_{r}=$ $55 \times 10^{3}$. Both PDSs accelerated collagen matrix reconstruction, and were possibly incorporated into the reconstructed collagen matrix. However, the effect of PG-I on the rate of collagen matrix reconstruction was much greater than that of $\mathrm{PG}-\mathrm{II}$ at an addition ratio of PDS of 1:500. Electron microscopy of reconstructed collagen matrices demonstrated that the collagen matrices consisted of native type fibrils and thin fibrils with indistinct banding patterns. Differential scanning calorimetry of the collagen matrix reconstructed with PG-I demonstrated that a great proportion of the components denatured at low temperatures compared with that with PG-II. It is difficult to explain the relationship between the structure of PDSs from PG-I and PG-II and the differences in the effect on the aggregation and matrix reconstruction of collagen molecule, because both PDSs contained two components with different mobilities on SDS-PAGE. However, since both preparations have given bands with the same migrations, it is considered that those PDSs differ from each other merely in relative contents of the two components with different mobilities.

\section{References}

1) K. Shirai, I. Ikenoue, K. Wada and A. Kawamura, Jpn. J. Zootech. Sci., 50, 217 (1979).

2) K. Shirai, K. Wada and A. Kawamura, Jpn. J. Zootech. Sci, 50, 223 (1979).

3) K. Shirai, k. Wada and A. Kawamura, Agric. Biol. Chem., 43, 2045 (1979).

4) K. Shirai and K. Wada, Das Leder, 34, 197 (1983).

5) K. Wada and K. Shirai, Proceedings the Vth World Conference on Animal Production, Vol. 2, 1983, p. 743.

6) K. Takahashi, K. Shirai and K. Wada, Jpn. J. Zootech. Sci., 59, 186 (1988).

7) K. Shirai and K. Wada Jpn. J. Zootech. Sci., 52, 749 (1981).

8) K. Takahashi, K. Shirai and K. Wada, Communications of XIX Congress of International Union of Leather Technologists and Chemists Societies, 1987, p 11.

9) S. P. Damle, L. Coster and J. D. Gregory, J. Biol. 
Chem., 257, 5523 (1982).

10) S. P. Damle, F. J. Kieras, W-K. Tzeng and J. D. Gregory, J. Biol. Chem., 254, 1614 (1979).

11) A. R. Poole, Biochem. J., 236, 1 (1986).

12) N. Fujii and Y. Nagai, J. Biochem., 90, 1249 (1981).

13) T. Nakamura, E. Matsunaga, H. Shinkai, Biochem. $J ., 213,289$ (1983).

14) I. Miyamoto and S. Nagase, J. Biochem., 88, 1793 (1980).

15) J. M. Snowden and D. A. Swann, Biopolymers, 19, 767 (1980).

16) K. G. Vogel, M. Paulsson and D. Heinegard, Biochem. J., 223, 587 (1984).

17) J. E. Scott and M. Haigh, Biosci. Rep., 5, 71 (1985).

18) J. E. Scott, Biochem. J., 252, 313 (1988).

19) C. A. Antonopoulos, I. Axelsson, D. Heinegard and S. Gard, Biochim. Biophys. Acta, 338, 108 (1974).

20) T. Bitter and H. M. Muir, Anal. Biochem., 4, 330 (1964).

21) Y. Ninomiya, R. Hata, Y. Nagai, S. Tajima, T. Nishikawa and H. Hatano, Biomedical Res., 3, 70 (1982).

22) R. Hata and Y. Nagai, Anal. Biochem., 45, 462
(1972).

23) Y. Oike, K. Kimata, T. Shinomura and S. Suzuki, Biochem. J., 191, 203 (1980).

24) K. Weber and M. Osborn, J. Biol. Chem., 244, 4406 (1969).

25) K. Takahashi, K. Yoshimura, K. Shirai and K. Wada, Jpn. J. Zootech. Sci., 57, 497 (1986).

26) R. F. Itzhaki and D. M. Gill, Anal. Biochem., 9, 401 (1964).

27) B. Obrink, Biochim. Biophys. Acta, 264, 354 (1972).

28) H. Yamanishi and Y. Sato, J. Biochem., 79, 131 (1976)

29) P. L. Privalov and E. I. Tiktopulo, Biopolymers, 9 , 127 (1970).

30) K. Takahashi, N. Awazu, K. Shirai and K. Wada, Jpn. J. Zootech. Sci., 58, 664 (1987).

31) D. G. Wallace, R. A. Condell, J. W. Donovan, A. Pairinen, W. M. Rhee and S. B. Wade, Biopolymers, 25, 1875 (1986).

32) J. M. Mcpherson, S. J. Swamura, R. A. Condell, W. Rhee and D. G. Wallac, Collagen Rel. Res., 1, 65 (1988). 